\title{
Inclusion of window opening habits in a window model based on activity and occupancy patterns
}

\author{
Silke Verbruggen ${ }^{1, *}$, Marc Delghust ${ }^{1}$, Jelle Laverge ${ }^{1}$, and Arnold Janssens ${ }^{1}$ \\ ${ }^{1}$ Ghent University, Faculty of Engineering and Architecture, Research Group Building Physics, 9000 Gent, Belgium
}

\begin{abstract}
The occupants' window opening behaviour can have a substantial influence on the indoor climate and the energy use in low energy dwellings. In literature, most window opening models are based on outdoor and/or indoor climate variables. However a study of Verbruggen et al. [1] revealed that these models are not able to predict the window opening behaviour accurately in wintertime, which may be attributed to the presence of window opening habits. The occupants perform the habits not according to a fixed time step but rather to the performance of a reoccurring activity or an occupancy change. Therefore, a window opening model is generated based on the occupancy and activity patterns of the inhabitants. The model links certain behaviours to specific activities or moments in an occupant's day without relating it to an exact time-step or specific weather conditions. Data on these habits and the links with occupancy are acquired from a survey conducted in a NZEB case-study project in Belgium. This paper includes the results of the habit-survey and explains how the window use model based on habits is generated. Based on the answers from the survey the window use in bedrooms and bathrooms could be fully defined for $93 \%$ of the households, only in the living room no complete window use profile could be defined. The developed model is able to predict the window use in a more realistic way compared to weather-models, with window opening actions linked to specific moments in the occupant's day.
\end{abstract}

\section{Introduction}

In recent years, climate change awareness became more important and with that the need to conserve energy. This led to an evolution in the building sector to (nearly) zeroenergy buildings. These energy-efficient buildings have lower transmission and infiltration heat losses, as well as lower ventilation losses due to more efficient ventilation systems. This implies that the energy losses due to airing get increasingly important with more energy efficient buildings. Nevertheless, window opening behaviour is generally neglected in energy simulations. In the past, however, some window opening models have been developed to estimate the associated ventilation losses.

Some of these models were simple models that predicted the window opening characteristics based on the analysis of a large sample of the building stock and the assumption that the occupants of other dwellings will act in the same way [2], [3]. These models were purely based on the observations in the case studies; consequently, they predicted the window opening behaviour for a specific context, occupant and time.

In the majority of studies, window opening behaviour was statistically defined based on weather and indoor climate parameters [4]-[14]. Almost all models included the outdoor temperature; however, there was no agreement on the significance of other weather variables (wind velocity, solar irradiation, precipitation, indoor temperature, relative humidity, and $\mathrm{CO}_{2}$ ). Some of these models predicted the duration of window opening in a specific period [2], [4], others predicted the number/proportion of rooms in which the windows were opened [5]-[9], [14]. These models were not specific enough to generate daily window opening profiles that included the variations in behaviour during the day. Recently, more detailed models were created that predicted the probability that a window is open [10]-[12] or will be opened [13]. Most of these models only included weather variables, however, some [4], [8]-[10], [13] included the time of day as well.

Nevertheless, the study of Verbruggen et al.[1] on the window opening behaviour of social housing tenants in a Belgian case study revealed that the weather variables could not accurately predict the window opening behaviour in wintertime. The weather variables had a significant influence on the window opening behaviour when yearly data were considered; however, the influence was not significant when only winter-data were analysed. Almost $42 \%$ of the occupants questioned in that study demonstrated some kind of window opening habit [15]. A window opening habit was defined as: "an action with a window that is repeated daily around the same time independently of the prevailing weather conditions". These habits were assumed to be the cause for the missing correlations between the weather variables and the window use in wintertime. The habits were sometimes

\footnotetext{
*Corresponding author: silke.verbruggen@ugent.be
} 
repeated every day at exactly the same time, however often the opening action shifted slightly in time. Therefore, it was assumed that occupants perform the habits not according to a fixed timestamp but rather to reoccurring activity and/or occupancy patterns. For example, the bedroom habits in the morning were probably linked to the waking-up event of the occupants, not to an exact time.

The goal of this study is to create a window use model that reflects the real window use in different households. The inclusion of window opening habits in the model is therefore necessary. This is challenging, since habits are very personal and vary greatly among the different occupants. In this paper, we first explain the methods used to gather information on the habits. Subsequently, the results of the survey are discussed in detail. Finally, it is clarified how the window use model that incorporates these habits is created and the model is compared to a weather-based model.

\section{Methods}

The study was based on two sets of data. The first data set encompasses the data used in the study of Verbruggen et al. [1], [15]. Since the study was not specifically designed to find out if the occupants were performing habits or linked their window use to specific activities, some of the window opening habits may not be detected, especially when the habit was not performed at the exact same time each day.

Therefore, a second survey was carried out in order to detect habits that are linked to certain activities or occupancy-states in the households. The questions were based on the findings from the first data-set. For example, it asked if the occupants perform any actions with the windows when they wake up as well as when they go to sleep, when they leave the house or when they cook. Most questions were asked for each room individually. Only the first two questions, related to the occupancy-state, focussed on the entire dwelling. The questions are given in Table 1.

The survey was conducted in August 2018 in a nearlyzero-energy social-housing neighbourhood in Kortrijk, Belgium [16] equipped with monitoring of the window states in some dwellings. The survey included 14 households. The case-study was the same as in the study of Verbruggen et al.[1], [15]; however, other households were queried.

Table 1. Questions posed in the survey.

\begin{tabular}{|l|}
\hline Do you change the state of the windows when you: \\
Leave the dwelling? \\
\hline Go to sleep? \\
\hline Wake-up? \\
\hline Cook? \\
\hline Clean? \\
\hline Smoke? (if applicable) \\
\hline Perform other activities? Which? \\
\hline Do you have any specific habits during summer? \\
\hline Do you have any specific habits during winter? \\
\hline
\end{tabular}

\section{Results}

\subsection{Window opening habits linked to activity and occupancy}

The goal of the study was to link window habits to specific occupancy and activity patterns. Taking occupancy into consideration in window opening models is necessary since occupants can only interact with their windows when they are at home and awake. During the night or times of absence, the state of the window will not be changed. Furthermore, most occupants will not evaluate their comfort every minute, and consequently not change the window state every few minutes. They rather adapt the state of the windows when the activity or occupancy-state is changed or after a longer period. This is often forgotten in the weather-based models. Although some models include time of day as a variable [8]-[10] or create different models for daytime and nighttime [4], [13], the possibility remains that the windows will be opened at times that the occupant is not able or willing to do so. From the study of Verbruggen et al. it was revealed that specific activities, such as cleaning or cooking, lead to specific opening behaviour as well. In this way, the window habits are not time dependent but rather activity dependent.

This requires a revision of the definition of a window opening habit, as given in the introduction. It was defined as: "an action with a window that is repeated daily around the same time independently of the prevailing weather conditions". This definition is strictly time focussed, therefore we return to the definition of a habit as defined by Ouelette and Wood in 1998 [17]: "A habit is an automatic action carried out without conscious effort that is the consequence of frequently repeating this action in a stable context." This definition specifies that the habit is carried out without thinking about it, so without evaluating the weather or other contextual variables. Further, the habit is only performed in a stable context, indicating that the context in which the habit is performed is identical each day. When this is not the case, the habit might not be performed. For example, an occupant may have the habit of opening the living room window every morning when he wakes up and leave it open until lunchtime. He will repeat this every day without consciously thinking about it or evaluating why he does it. If he gets a visit in the morning from a friend, he might decide to close the window earlier since that friend is not comfortable with the open window. The visiting friend is a change in context, which leads to the termination of the habit. In this way, a window opening habit can be seen as: "an action with the window that is repeated every day before, after or during the execution of certain activities, in the stable situation of everyday life".

The questions asked in the survey to detect these habits could be divided in three categories: occupancyrelated questions, activity-related questions and roomrelated questions. 


\subsubsection{Window use in relation to occupancy-state}

The first questions were posed in order to detect window opening habits that are related to the occupancy-state of the inhabitant. The inhabitant can be either home and awake, home and asleep or away from home. During the periods of inactivity (sleep or away), the inhabitant is not able to interact with the windows. Some occupants prefer to sleep with the window open in the bedroom, while others always close it. Some occupants may close the window when leaving the dwelling for security reasons, but if this is not a problem, the window may remain open during their absence.

The results from the survey showed a great variation in window opening behaviour between different households. Half of the occupants responded that they keep the windows in the current state when leaving the dwelling, while $29 \%$ always close all windows and $21 \%$ only close the accessible windows for safety reasons. In correspondence to this, $57 \%$ of the occupants leave the windows in the current state when going to bed, while $36 \%$ close all windows and $7 \%$ only close the bedroom window. It can be concluded that for approximately half of the occupants the window opening behaviour is defined during periods of absence and during the night. For the other occupants the window state is dependent on the state just before going to sleep or before leaving the dwelling.

\subsubsection{Window use in relation to residential activities}

Based on the study of Verbruggen et al.[15] it was expected that a large part of the occupants would open the windows when waking up. From the question "Do you change the state of your window when you wake up?" it was revealed that $43 \%$ of the occupants open one or more windows when waking up. All of these occupants opened at least one window in the bedroom, only one of the occupants responded also opening a window in the living room.

The other questions concerned household activities such as cleaning and cooking. For cleaning, no distinct habits were detected, since only one occupant opened the windows while cleaning. Cleaning can encompass many different types of activities (vacuuming, dusting, mopping,...) consequently, it is difficult to give a general type of window use during this activity. It is expected that most occupants open their windows while cleaning them.

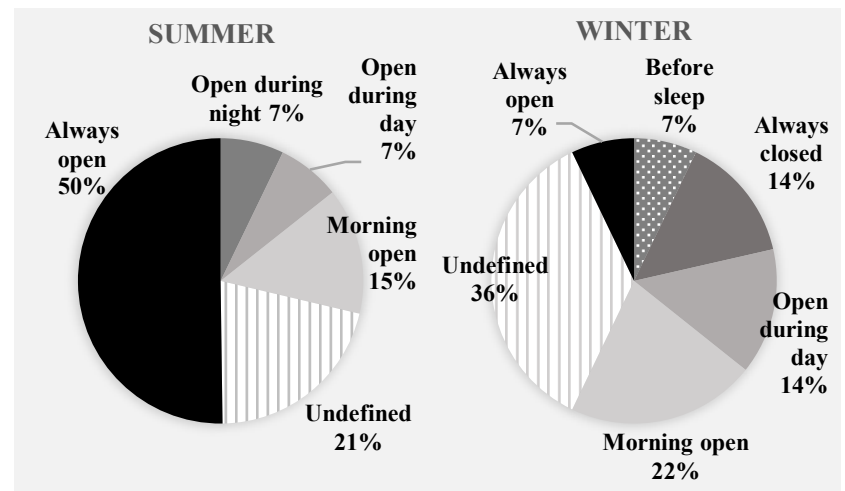

Therefore, it should be considered to ask specific questions about sub-activities of cleaning.

$29 \%$ of the occupants open a window while cooking, although all respondents dispose of a cooker hood. Either they preferred opening the window over using the cooker hood or they used both simultaneously. Only three occupants smoke regularly, and only one of them opens a window for this reason. The others go outside. No other activities were mentioned that related to window use. However, there may still be other habits present since occupants do not consciously think about the habits and consequently, they might fail to mention them.

\subsubsection{Window use in different rooms in winter and summer}

During the performance of previous studies [1], [15], we noticed that many people said: "in that room, at that moment, I open the window". Therefore, we also included the questions "During winter/summer at what moment do you open and close your windows in the living room/bedroom/bathroom?". Not all occupants could of course respond to these questions since their window opening behaviour varied, however, many occupants could give some general insights in the window use in those rooms.

Most occupants had a clear view on the window use in their bedrooms. The results for summer and winter are given in Figure 1 (left). In summertime, $50 \%$ of the occupants leave the windows always open, while $7 \%$ leave the windows only open when they are sleeping in the room, while another $7 \%$ do just the opposite by keeping the window open during the day but not during the night. $15 \%$ expressed that they open the window in the morning when they wake up, and leave it open for a predetermined time, often 1 hour. Only $21 \%$ could not define a general trend. In wintertime, the proportion of occupants that leave the windows always open decreased significantly. However, there is still one occupant (7\%) that leaves the bedroom window continuously open during wintertime when the temperatures outside can drop below freezing point. Only $14 \%$ mentioned that they never open their window in wintertime, all other occupants open the windows still frequently, with $14 \%$ that leave the window open during the day when they are not in the room, $22 \%$ that open the window in the morning for a predetermined time and $7 \%$ that open the windows

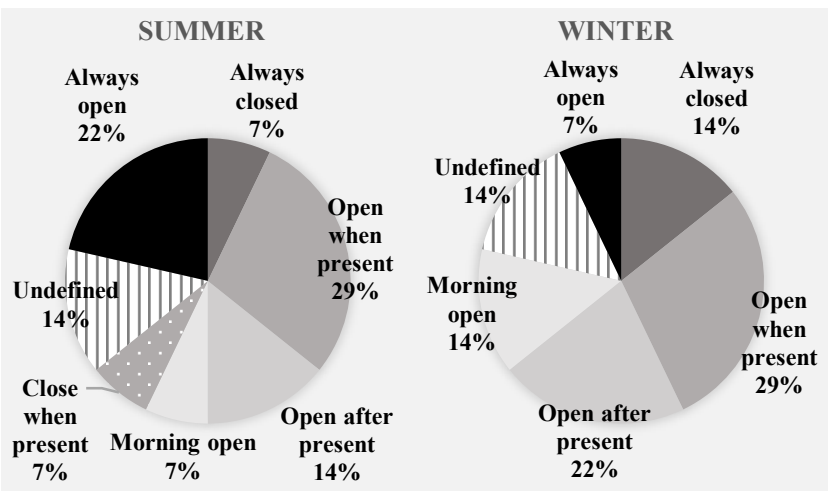

Figure 1. Window use in wintertime and summertime in the bedroom (left) and bathroom (right). 
one hour before going to bed. The proportion of occupants that could not define a specific window use increased slightly to $36 \%$ in comparison with summertime.

Most occupants could as well define how they interact with the bathroom windows (Figure 1 - right). In the bathroom, the window use is closely related to the presence in this room. In both summer and winter, 29\% open the window when they are present in the bathroom. Another $14 \%$ and $22 \%$ in winter and summer respectively, open the windows after they were present in the bathroom. In summer, one occupant closes the window when present in the bathroom; this is related to privacy issues. Further, in summer 22\% leave the windows always open, while in winter one occupant still leaves the window always open. One occupant never opens the bathroom window. It can be concluded for the bathroom that the use of the room has a high impact on the window opening behaviour, this may be related to the accumulation of moist (even though a ventilation system is present in all houses).

In the living room, the window use could not be defined by half of the occupants for summertime and even $71 \%$ for wintertime. In summertime, $43 \%$ of the occupants leave the windows open, while in one household the windows are only open during the night. In wintertime, the remaining $29 \%$ never open their windows. In the living room, the room-habits are less defined compared to in the bedroom and bathroom.

\subsubsection{Window use profiles based on survey responses}

Based on the answers to these few questions, it was possible to derive general window opening profiles for most of the households. The information gathered from the questions related to the occupancy-state can be used to determine the window opening actions before the occupants go to sleep or leave the dwelling. Additionally, the specific room-habits make it possible to clearly define the window use in some rooms. Next, activity-habits performed during the day lead to a more detailed window use profile, adding extra moments that the window will be opened or closed, sometimes overriding the in the previous steps defined patterns. For example, if an occupant leaves the living room window always closed, but has the habit to open the window while cleaning; this cleaning-habit will override the general room-habit. After these steps, some periods during the day may remain undefined. On these moments an extra window opening model is needed, which may be based on weather variables.

An example of a window use profile in wintertime is given in Figure 2. The profile is evidently not related to timestamps, so no hourly values are given on the $\mathrm{x}$-axis. For graphical purposes, the profile is indicated by symbols referring to the activities. In the example, the occupants indicated that they close all windows when they go to sleep. Further, they mentioned that during the use of the bathroom the window was opened and that the window in the bedroom was opened when they were not present in the room. In the living room/kitchen, the

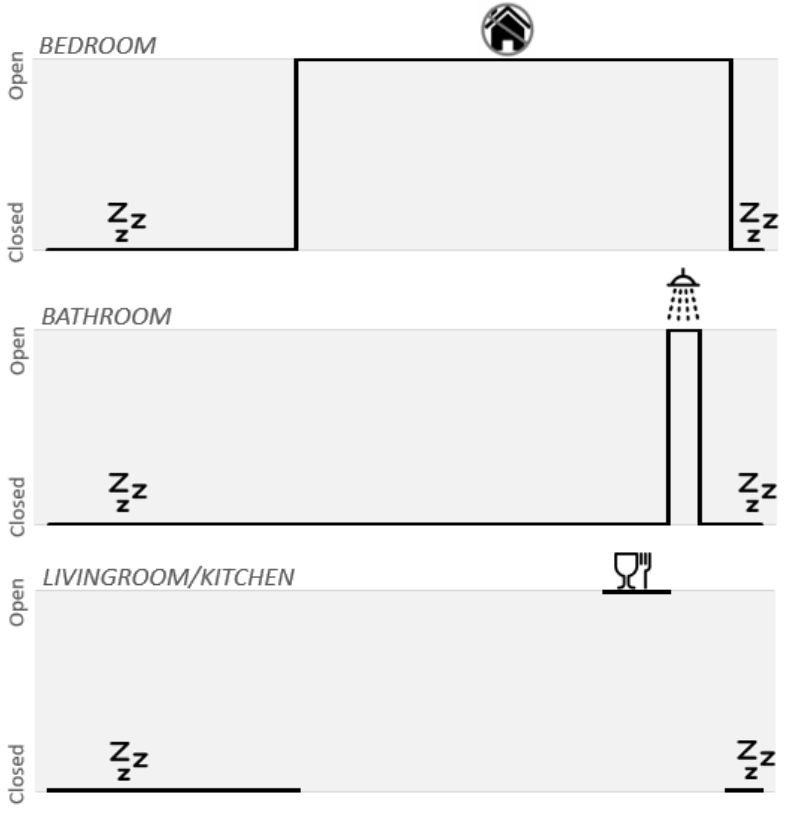

Figure 2. Example of a window use profile in winter.

window was opened during cooking but no extra information was given. With this information, a complete window use profile could be made for the bedrooms and the bathroom. In the living room, the window use during a part of the day remained undefined; consequently, an additional window use model is needed to define the window state at those moments.

For $28 \%$ of the households a complete window use profile for summer and winter could be generated based on the responses to the survey. This relatively low percentage is caused by the lack of defined habits in the living room. In the bedroom and bathrooms, the window use profiles could be fully generated for $93 \%$ of the households. These results support the motivation to create a window use model based on habits, specifically for the bedrooms and bathrooms.

\subsection{Preliminary window use model}

The creation of these window use profiles can be generalised to a window opening model. This model will be able to predict a large part of the window opening behaviour of households by their habits based on their occupancy and activity profiles.

\subsubsection{Stochastic window use model}

From the responses to the survey, an initial stochastic window use model is created. It should be remarked that this is based on a small data-sample and therefore more studies will need to be done in order to get a statistically significant model. The scheme of the model is given in Figure 3.

In the first step the occupancy and activity profiles of the household are determined by an occupancy and activity prediction model such as, for example, the StROBe-model [18]. 
Next, it is determined which occupancy-habits are performed by comparing the probability of having a specific habit to a random number. The probabilities are derived from the survey results. In this way, the window states while the occupants are away or asleep could be determined for some of the households.

Based on the occupancy-habits the possible options of the room-habits are restricted. For example, if the occupants always close the windows when going to sleep, they will not be able to belong to the room-habit of 'always leaving the bedroom windows open'. Based on the adjusted possibilities, due to these restrictions, it is determined which room-habit the household performs. According to the selected habits, the window states in the rooms are defined.

Next, it is determined if the household performs an activity-habit, again based on statistics from the survey. If a habit is present, the habit will be linked to the specific activity profile and linked to the room in which the activity is performed. The window states generated in this step can overrule the previous determined window states, as discussed before. As a last step, it is checked if there are moments in the simulated period when not all window states are determined. If this is the case, an additional window use model will be applied to determine the state of the windows on these moments.

\subsubsection{Comparison with other window-use models}

The habit-model is compared to a weather-based window use model. The model of Maeyens and Janssens [10] was chosen for this task. It describes the probability of opening a window every hour based on the outdoor temperature, wind velocity and solar irradiation. A correction factor (Francastoro) is applied to compensate for the presence of the occupants; this factor is based on the hourly average presence. Measured data of a climate station in Kortrijk [1] are used as input, this for one summer day and one winter day. Since the model of Maeyens et al. does not differentiate between the different rooms, similar profiles will be generated for all rooms.

A resulting window use profile is given in Figure 4. In this room, two opening actions (120 min) occur during the winter day. In summer more actions occur, resulting in a total opening time of 480 minutes.

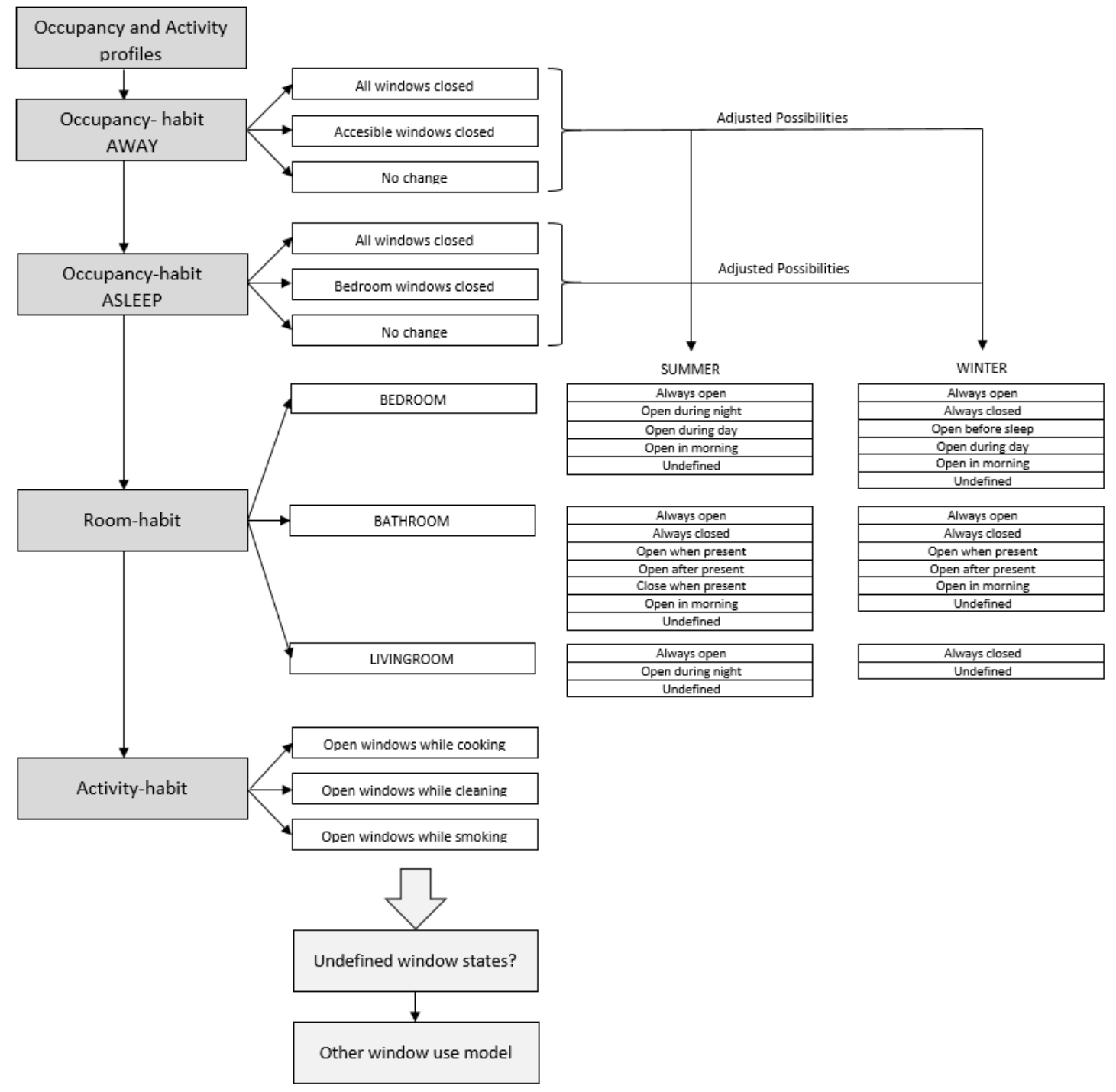

Figure 3. Window use model based on occupancy and activity states. 
In the habit-model a differentiation has been made between the different rooms. Likewise, the model is simulated for one summer day and one winter day. The occupancy and activity patterns are kept the same for the two days. Figure 5 shows the occupancy-state and the resulting window use profiles.

The generated household leaves the windows as they are when they go to bed or leave the dwelling. In wintertime, the bathroom window is only opened when the occupants are present, which leads to an opening time of 30 minutes. In the bedroom, on the other hand, the window is opened during the day when the occupants are not present in the room (960 $\mathrm{min})$. No room-habit was defined for the living room; therefore, the weather model of Maeyens et al. is applied during these undefined periods. Additionally, a cooking-habit is present. This leads to a total opening time of 80 minutes. In summertime, the living room window is always opened. While the room-habits in the bedroom and bathroom are undefined, leading to low opening times of 30 and 60 minutes respectively.

The window use profile generated by the habit-model is more realistic, since the opening actions happen more logically in the occupant's day. All actions happen when the occupants are effectively at home and active, which is not the case in the weather-model profile where for example every hour during the summer night a window opening action happens.

Since the models are stochastic, we are not able to compare the opening durations of this one simulation, as it may represent an extreme window use profile. Therefore, the simulation is repeated hundred times for the specific winter and summer day. Additionally, these two models are compared to measured data. This data is gathered from 10 houses in the same neighbourhood, where window sensors were installed. The households are not the same as the ones from the survey.

In wintertime, the bathroom (106 $\mathrm{min}$ ) and bedroom (205 min) windows are on average opened more with the habit-model in comparison to the weather-model (34 min) (Figure 6). This can be attributed to the fact that in the bathroom and bedrooms the most habits are present, which lead to longer opening times in winter. The habitmodel is in good comparison with the measured data, with almost the same average opening duration for the bedroom (196 min) and a slightly higher opening duration (144 $\mathrm{min})$ for the bathroom. In the living room the opening time of the habit-model is a bit lower (19 $\mathrm{min})$ compared to the weather-model, which is similar to the measured data. From the results, we can conclude that the window opening behaviour in the bedrooms and bathroom can be quite accurately predicted by the habitmodel, however, in the living room the habit-model and weather-model generate similar average durations.

In summertime, the average opening duration of the bathroom with the habit-model (378 min) is quite similar as in the model of Maeyens (418 min), but lower compared to measured data (599 $\mathrm{min})$. In the bedroom and living room, the models predict different opening durations, with the weather-model severely underestimating the opening time.
The habit-model includes more variation in window use between different simulations. Where the weathermodel only predicts opening durations in winter from 0 to 180 minutes, the habit-model predicts opening durations from 0 to 1440 minutes in the bedroom and bathroom, which is similar to the measured data. These larger variations are the results of the different extreme habits, such as leaving the windows always open or leaving them always closed, which are present in everyday life.

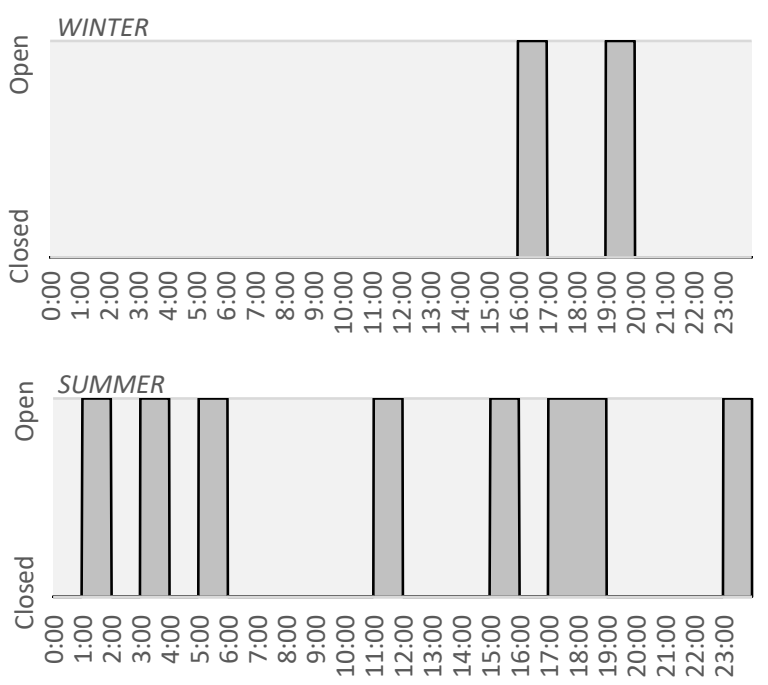

Figure 4. Window use profiles according to the window use model of Maeyens et al.

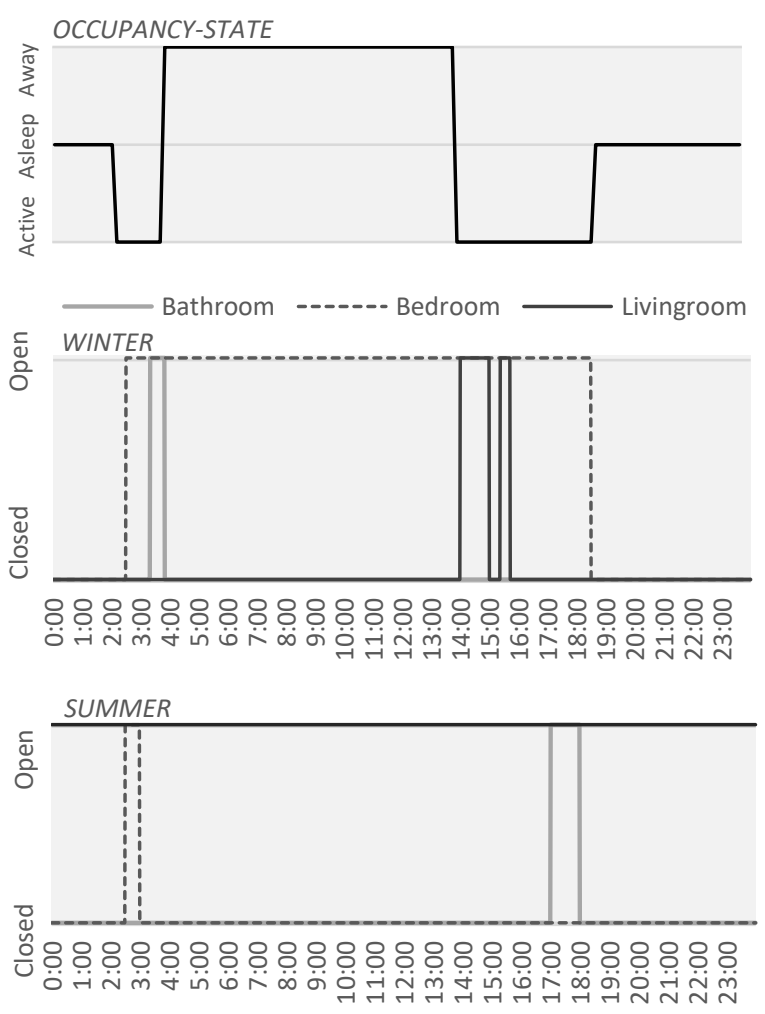

Figure 5. Occupancy-state (top) and window use profiles (bottom) according to the window use model based on habits 


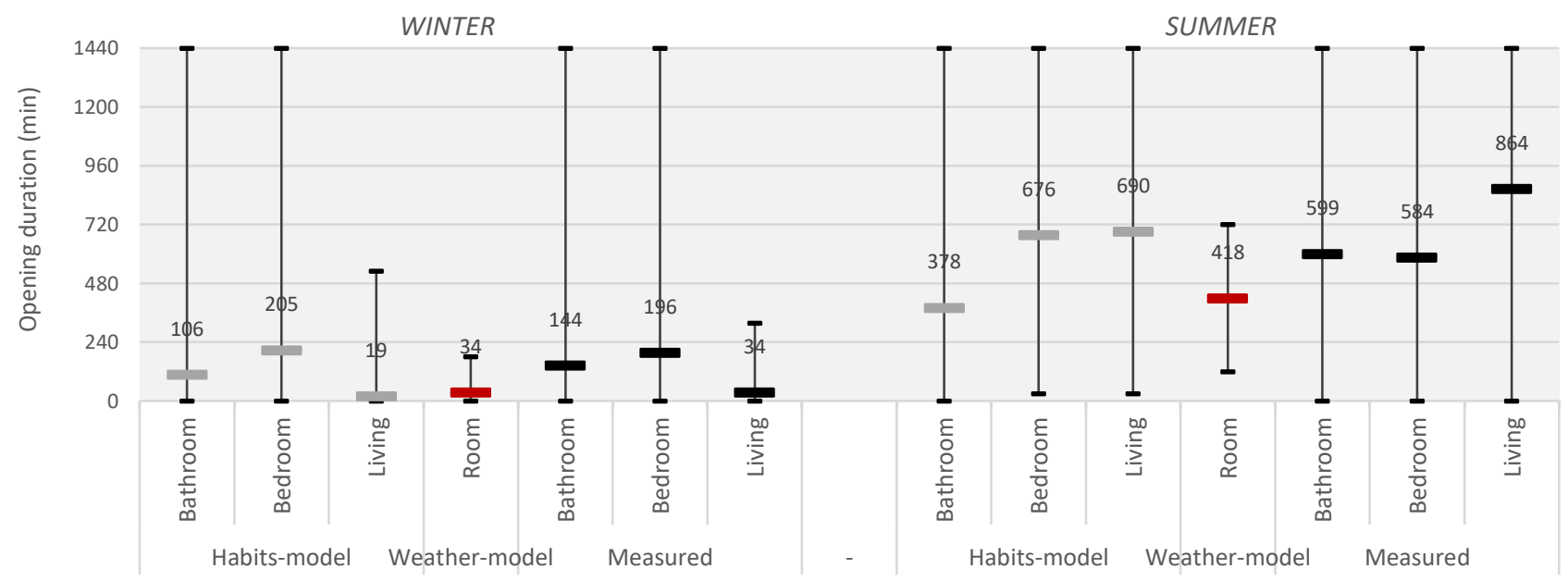

Figure 6. Minimum, maximum and average opening durations for the different rooms with the habit-model, the weather model and measured data.

\subsubsection{Remarks on the window use model}

Some essential remarks should be made on this window use model. First, the statistics used in the model are derived from a very small data-sample of 14 households. This jeopardizes the significance of the model. In order to solve this problem an online survey is currently under development to be send out to a larger sample group.

Secondly, the model predicts the window opening behaviour randomly for each household. However, it is assumed that occupants in a household/dwelling with specific characteristics are more likely to perform one habit over the other. Therefore, the online survey will as well be used to link these characteristics to the habits.

Thirdly, the model only creates window use profiles for wintertime and summertime. However, at a certain moment the winter-habits will dissipate and change in summer-habits. It is for now unclear at which moment this happens, as it will probably also differ between households. It is as well indeterminate if the winter-habits change directly in summer-habits or if there are some inbetween-season-habits (spring/autumn-habits).

A fourth and last remark should be made concerning the type of habits questioned. The habits that are questioned are derived from the results of two surveys in a social housing NZEB-neighbourhood, consequently focussing on very specific households and dwellingtypes. Other habits may be present in other households or other types of dwellings. Again, broadening the survey sample size will help to detect more habits.

\section{Conclusion}

The window opening model based on habits which is developed in this study, creates more realistic window use profiles compared to models based on weather-variables. The model only allows for window opening actions when the occupants are active and at home, and the opening actions occur closely related to events in the occupant's day, such as specific activities or changes in occupancystate.
The model generates window use profiles based on statistics. Consequently, large variations in opening durations are present, which is the case for the measured data as well. In order to determine window use profiles for a specific family, their specific habits need to be known. These habits can be detected by asking only a few basic questions (see Table 1). If the habits are known, an accurate window use profile can be generated for most households.

Focussing further research on the links between habits and specific household or dwelling characteristics will allow for an accurate window use model that has no need for habit-questionnaires. Additional research is needed as well for determining other types of habits that may have been missed in this study. Further, the survival time of the winter-habits and summer-habits needs to be determined, in order to generate detailed yearly window use profiles instead of daily profiles.

We gratefully acknowledge the financial support received for this work from the Fund for Scientific Research (FWO) in the frame of the strategic basic research (SBO) project "NEPBC: Next generation building energy assessment methods towards a carbon neutral building stock" (S009617N). The authors would also like to thank the social housing company 'Goedkope Woning' and the occupants who willingly participated in this project.

\section{References}

[1] S. Verbruggen, A. Janssens, J. Laverge, M. Delghust, Window opening behavior in a CO2neutral neighborhood (Ghent University, 2017)

[3] C. Dubrul, Inhabitant Behaviour with Respect to Ventilation (1988)

[4] H. Erhorn, Energy Build. 11, 267-275 (1988)

[5] B. Jeong, J. W. Jeong, J. S. Park, Energy Build. 127, 206-216 (2016) 
[6] G. W. Brundrett, Int. J. Energy Res. 1, 289-298 (1977)

[7] G. W. Brundrett, Proc. of 1st W.H.O. Int. Indoor Climate Symp. (1978)

[8] G. Conan, Domestic gas consumption, household behaviour patterns, and window opening (Brunel University, 1981)

[9] G. Conan, Energy efficient domestic ventilation systems for achieving acceptable indoor air quality, 3rd AIC conference (1982)

[10] J. Maeyens, A. Janssens, Proc. of 2th IBPC, 737747 (2003)

[11] H. B. Rijal, P. Tuohy, M. A. Humphreys, J. F. Nicol, A. Samuel, J. Clarke, Energy Build. 39, 823-836 (2007)

[12] P. Tuohy, H. B. Rijal, M. a Humphreys, J. F. Nicol, A. Samuel, J. Clarke, Proc. of Building simulation 717-724 (2007)

[13] R. Andersen, Occupant behaviour with regard to control of the indoor environment (Technical University of Denmark, 2009)

[14] M. D. Lyberg, Residents and windows, 2 (1983).

[15] S. Verbruggen, M. Delghust, J. Laverge, A. Janssens, Proc. of 7th IBPC, 677-682 (2018)

[16] E. Himpe, A. Janssens, J. E. V. Rebollar, Energy Procedia 78, 3465-3470 (2015)

[17] J. A. Ouellette, W. Wood, Psychol. Bull. 124, 54-74 (1998)

[18] R. Baetens, D. Saelens, J. Build. Perform. Simul., 9, 431-447 (2016). 